Pacific Journal of Mathematics

SCHUR'S THEOREM AND THE DRAZIN INVERSE 


\title{
SCHUR'S THEOREM AND THE DRAZIN INVERSE
}

\author{
ROBERT E. HARTWIG
}

It is shown that if $M=\left[\begin{array}{ll}A & C \\ B & D\end{array}\right]$ is a square $2 n \times 2 n$ matrix over a ring $R$, such that $A C=C A \in R_{n \times n}$, and with the property that $A$ and $C$ possess Drazin inverses, then $M$ is invertible in $R_{2 n \times 2 n}$ if and only if $D A-B C$ is invertible in $R_{n \times n}$.

1. Introduction. In a recent paper [7], Herstein and Small extended the classic result of Schur [5, p. 46] to matrices over $E$-rings. These are rings for which every primitive image is artinian. This result states that for a square complex block matrix $M=\left[\begin{array}{ll}A & C \\ B & D\end{array}\right]$, with $A, B, C, D$ square of the same size such that $A C=C A$, then $M$ is invertible exactly when $\Delta=D A-B C$ in invertible. This is a different but equivalent formulation of the problem as stated in [7].

The purpose of this note is to show that this result by Schur is basically a consequence of the local existence of the Drazin inverse [2] of the matrices $A$ and $C$; that is, the strong- $\pi$-regularity of $A$ and $C$ [1] [4]. The proof of [7] was based on the fact that Schur's result for matrices over $E$-rings is really equivalent to the corresponding result for matrices over simple artinian rings (which may be taken to be division rings). Since artinian rings with unity are noetherian [8], p. 69, it follows that artinian rings with unity are strongly- $\pi$-regular, so that our local result extends the Schur theorem for artinian rings as proven in [7].

The Drazin inverse $a^{d}$ of a ring element $a$, is the unique solution, if any, to the equations

$$
a^{k} x a=a^{k}, x a x=x, a x=x a,
$$

for some $k \geqq 0$, while the group inverse $a^{\sharp}$ of $a$ is the unique solution, if any, of these equations with $k=0$, or 1 . For example, if $a$ is algebraic over some field $\mathscr{F}$ and $a^{n+1} b=a^{n}$, with $a b=b a$, then $a^{d}=$ $a^{n} b^{n+1}$. The element $a^{d}$ exists if and only if $a$ is strongly- $\pi$-regular, that is, when both chains $\left\{a^{i} R\right\}$ and $\left\{R a^{i}\right\}$ are ultimately stationary, [5, Theorem 4]. A ring element is called (von Neumann) regular if $a a^{-} a=a$ for some ring element $a^{-}$. If there exists such $a^{-}$that is invertible, $a$ is called unit-regular.

We shall assume familiarity with the properties of these inverses [4] [2] [6] and in particular with the fact that $a c=c a \Rightarrow a^{d} c=c a^{d}$ [4, Theorem 1].

It is known that, unlike regularity and unit regularity, $R_{2 \times 2}$ does 
not inherit strong-regularity from $R$ [9] [11]. It is not known however, whether the strong- $\Pi$-regularity of $R$, or the related concept of finite regularity ( $a b=1 \Longrightarrow b a=1$ ) is inherited by $R_{2 \times 2}$ [10].

We shall use the notation ${ }^{\circ} S$ and $S^{0}$ to indicate the right and left annihilators of $S$ respectively, e.g.,

$$
S^{0}=\{x \in R ; x s=0, \forall s \in S\} .
$$

For notational convenience we shall state our results in terms of rings $R$ with unity, with the translation to matrices over $R$, being self evident. In particular $a R+c R=R$ is equivalent to the $1 \times 2$ matrix $[a, c]$ having a right inverse.

2. Preliminaries. The key to our main result are the following two lemmas.

LEMMA 1. Let $R$ be a ring with unity 1 , and let $e, f$ be commuting idempotents in $R$. If $g=e+f(1-e)$ then

(i) $g^{2}=g$, (ii) $e R+f R=g R$, (iii) $R e+R f=R g$, (iv) $e^{0} \cap f^{0}=$ $g^{0}$, (v) ${ }^{0} e \cap{ }^{0} f={ }^{0} g$, (vi) $e R+f R=R \Leftrightarrow g=1 \Leftrightarrow R e+R f=R \Leftrightarrow e^{0} \cap$ $f^{0}=(0) \Leftrightarrow{ }^{0} e \cap^{0} f=(0) \Leftrightarrow(1-e)(1-f)=0$.

LEMMA 2. Let $R$ be a ring with unity 1 , and let $a, c$ be commuting elements of $R$. Then

(i) $a R+c R=R \Leftrightarrow a^{m} R+c^{n} R=R$ for some $m, n \geqq 1 \Leftrightarrow a^{m} R+$ $c^{n} R=R$ for all $m, n \geqq 1$.

(ii) ${ }^{0} a \cap{ }^{0} c=(0) \Leftrightarrow^{0}\left(a^{m}\right) \cap{ }^{0}\left(c^{n}\right)=(0)$ for some $m, n \geqq 1 \Leftrightarrow^{0}\left(a^{m}\right) \cap$ ${ }^{\circ}\left(c^{n}\right)=(0)$ for all $m, n \geqq 1$.

(iii) $R a+R c=R \Leftrightarrow R a^{m}+R c^{n}=R$ for some $m, n \geqq 1 \Leftrightarrow R a^{m}+$ $R c^{n}=R$ for all $m, n \geqq 1$.

(iv) $a^{0} \cap c^{0}=(0) \Leftrightarrow\left(a^{m}\right)^{\wedge} \cap\left(c^{n}\right)^{0}=(0)$ for some $m, n \geqq 1 \Leftrightarrow\left(a^{m}\right)^{0} \cap$ $\left(c^{n}\right)^{0}=(0)$ for all $m, n \geqq 1$.

If in addition, the Drazin inverses $a^{d}$ and $c^{d}$ exists, these conditions are all equivalent to

(v) $\left(1-a a^{d}\right)\left(1-c c^{d}\right)=0$.

Proof. The proof of (i)-(iv) follows by induction. Now suppose that $a^{d}$ and $c^{d}$ exist and that index $(a)=k$, index $(c)=l$. Then for all $m \geqq k, a^{m} R=a^{k} R=a^{d} R=a^{d} a R$. And so, taking $m \geqq k, n \geqq l$, we see that (i) is equivalent to

$$
R=a^{m} R+c^{n} R=a^{k} R+c^{l} R=a^{d} R+c^{d} R=a^{d} a R+c^{d} c R,
$$

which by Lemma 1 is equivalent to

$$
\left(1-a a^{d}\right)\left(1-c c^{d}\right)=0 \text {. }
$$


Left-right symmetry now shows that (iii) is also equivalent to (v). Lastly, since for $m \geqq k,\left(a^{m}\right)^{0}=\left(a^{k}\right)^{0}=\left(a^{d}\right)^{0}=\left(a^{d} a\right)^{0}$, it follows that with $m \geqq k, n \geqq l$, (iv) is equivalent to $\left(a^{d} a\right)^{0} \cap\left(c^{d} c\right)^{0}=(0)$, which again by Lemma 1 is equivalent to $(v)$. Symmetry again yields the remaining equivalence.

Before proceeding with our theorem we remark that:

1. It is not necessary for $a^{d}$ and $c^{d}$ to exist in order for

$$
R a+R c=R \Longleftrightarrow a^{0} \cap c^{0}=(0)
$$

to be valid. It would suffice if $a, c$ and $c\left(1-a^{-} a\right)$ were regular.

2. The equivalence of (iv) and ( $v$ ) has uses in the theory of differential equations, [2] Lemma 1. The above furnishes a short and purely algebraic proof of this useful result.

\section{Main results.}

THEOREM 1. Let $R$ be a ring with unity 1 and let $M=\left[\begin{array}{ll}a & c \\ b & d\end{array}\right] \epsilon$ $R_{2 \times 2}$ with $a c=c a$. Suppose further that $a^{d}$ and $\left[\left(1-a a^{d}\right) c\right]^{d}$ exist. If $\Delta=d a-b c$, then:

(i ) $\Delta$ is left invertible $\Leftrightarrow M$ is left invertible.

(ii) $M$ is right invertible $\Leftrightarrow \Delta$ is right invertible.

(iii) $M$ is invertible $\Leftrightarrow \Delta$ is invertible.

Proof. Consider the matrix

$$
N=\left[\begin{array}{ll}
a & u \\
b & z
\end{array}\right]=\left[\begin{array}{ll}
a & c \\
b & d
\end{array}\right]\left[\begin{array}{cc}
1 & -a^{d} c \\
0 & 1
\end{array}\right]
$$

where $u=\left(1-a a^{d}\right) c$ and $z=d-b a^{d} c$. Since $a, a^{d}$ and $c$ commute it follows that

$$
z a-b u=\left(d-b a^{d} c\right) a-b\left(1-a a^{d}\right) c=d a-b c=\Delta .
$$

Now because $a^{d} u=0=u a^{d}=a^{d} u^{d}=u^{d} a^{d}$, we may construct the matrices:

$$
\left[\begin{array}{ll}
a & u \\
b & z
\end{array}\right]\left[\begin{array}{rr}
a^{d} & -u \\
u^{d} & a
\end{array}\right]=\left[\begin{array}{cc}
a a^{d}+u u^{d} & 0 \\
t & \Delta
\end{array}\right]=T
$$

and

$$
\begin{gathered}
{\left[\begin{array}{cc}
a & u \\
-u^{d} & a^{d}
\end{array}\right]\left[\begin{array}{rr}
a^{d} & -u \\
u^{d} & a
\end{array}\right]=\left[\begin{array}{cc}
a a^{d}+u u^{d} & 0 \\
0 & a a^{d}+u u^{d}
\end{array}\right]} \\
\quad=\left[\begin{array}{rr}
a^{d} & -u \\
u^{d} & a
\end{array}\right]\left[\begin{array}{cc}
a & u \\
-u^{d} & a^{d}
\end{array}\right] .
\end{gathered}
$$


In general however, $a u^{d} \neq 0$ unless index $(a) \leqq 1$. Suppose now that $\Delta$ has a left inverse $\Delta^{-}$, then by (5),

$$
R=R a+R c=R a+R u .
$$

By Lemma 2, applid to $a$ and $u$, we see that

$$
\left(1-a a^{d}\right)\left(1-u u^{d}\right)=0
$$

or equivalently

$$
a a^{d}+u u^{d}=1 .
$$

Hence, by (7), it follows that the matrix $P=\left[\begin{array}{rr}a^{d} & -u \\ u^{d} & a\end{array}\right]$ is invertible. Now since

$$
\left[\begin{array}{cc}
1 & 0 \\
-\Delta^{-} t & \Delta^{-}
\end{array}\right]\left[\begin{array}{ll}
1 & 0 \\
t & \Delta
\end{array}\right]=\left[\begin{array}{ll}
1 & 0 \\
0 & 1
\end{array}\right]
$$

it follows that $M$ has a left inverse $M^{-}$and that

$$
R=R a+R b=R c+R d .
$$

If in addition $\Delta \Delta^{-}=1$, then

$$
\left[\begin{array}{ll}
1 & 0 \\
t & \Delta
\end{array}\right]\left[\begin{array}{cc}
1 & 0 \\
-\Delta^{-} t & \Delta^{-}
\end{array}\right]=I_{2}
$$

and consequently $M$ is also invertible.

Conversely, suppose that $M M^{-}=I$. Then because $N$ also has a right inverse, it follows that

$$
a R+u R=R .
$$

Again by Lemma 2, applied to $a$ and $u$, we may conclude that (9) holds so that $P$ is invertible. Hence $T=\left[\begin{array}{ll}1 & 0 \\ t & \Delta\end{array}\right]$ has a right inverse $T^{-}=$ $\left[\begin{array}{ll}\alpha & \gamma \\ \beta & \delta\end{array}\right]$. Now $T T^{-}=I \Rightarrow \gamma=0 \Rightarrow \Delta \delta=1$, and so $\Delta$ has a right inverse. If in addition, $M^{-} M=I$, then $T^{-} T=I$ and hence again as $\gamma=0$, $\delta \Delta=1$, completing the proof.

COROLlaRY 1. If $R$ is a ring with unity and $M=\left[\begin{array}{ll}a & c \\ b & d\end{array}\right] \in R_{2 \times 2}$ with $a c=c a$ such that $a^{d}$ and $c^{d}$ exist, then $M$ is invertible if and only if $\Delta=d a-b c$ is invertible.

Proof. Note that $a c=c a$ implies that $a a^{d} c=c a a^{d}$, so that $u^{d}=$ $\left(1-a a^{d}\right) c^{d}$. Again because square matrices over artinian ring with unity possess Drazin inverses, this result includes the second part of Theorem 2 of [7]. 
Corollary 2. Let $R$ be a ring with unity 1, and let $a, c \in R$ such that $a c=c a$ and $a^{d},\left[\left(1-a a^{d}\right) c\right]^{d}$ exist. Then if $R=R a+R c$ there exists $d \in R$ so that $\left[\begin{array}{ll}a & c \\ c & d\end{array}\right]$ is invertible.

Proof. From Theorem 1, it suffices to select $d \in R$ such that $\Delta=$ $d a-c^{2}$ is invertible. One such choice is given by $d=a^{d}+c^{2} a^{d}$, because then $\Delta=a a^{d}-u^{2}$ which has inverse $a a^{d}-u^{d} u^{d}$. Indeed, if $R=$ $R a+R c=R a+R u$, then $a a^{d}+u u^{d}=1$ which coupled with the fact $a^{d} u^{d}=0$, yields the desired result.

We conclude this note with several remarks.

1. If $a^{\sharp}$ exists we could also select $d=a+c^{2} a^{\sharp}$ in the last corollary, for then $\Delta=a^{2}-u^{2}$ has as inverse $\left(a^{\sharp}\right)^{2}-u u^{d}$ since now $a u=0$. Moreover, in this case

$$
\begin{aligned}
{\left[\begin{array}{cc}
a & c \\
c & a+c^{2} a
\end{array}\right]^{-1} } & =\left[\begin{array}{cc}
1 & -a^{\sharp} c \\
0 & 1
\end{array}\right]\left[\begin{array}{cc}
a & u^{d} \\
u^{d} & a
\end{array}\right]\left[\begin{array}{cc}
1 & 0 \\
-c a & 1
\end{array}\right] \\
& =\left[\begin{array}{cc}
a-\left(a^{\sharp}\right)^{3} c^{2} & u^{d}-c\left(a^{\sharp}\right)^{2} \\
u^{d}-c\left(a^{\sharp}\right)^{2} & a^{\sharp}
\end{array}\right] .
\end{aligned}
$$

2. The fact that: "If $a c=c a$, and $a^{d}$, $u^{d}$ exist, then $R=R a+R c$ ensures that $a^{d} a+u^{d} u=1$ ", should be compared with the corresponding results for Moore-Penrose inverses [6]. Namely, if $a^{\dagger}$ and $v^{\dagger}=$ $\left[c\left(1-a^{\dagger} a\right)\right]^{\dagger}$ exists, then

$$
R=R a+R c \Longrightarrow 1=a^{\dagger} a+v^{\dagger} v .
$$

3. If $a$ is unit-regular, that is $a a^{=} a=a$ for some unit $a^{=}$, then under suitable conditions $a R+c R=R \Rightarrow R a+R c=R$. Indeed if $u=\left(1-a a^{=}\right) c$ is regular and $c^{\sharp}$ exists, then

$$
a R+c R=R \Longrightarrow a a^{=}+\left(1-a^{=}\right) c u^{-}\left(1-a a^{=}\right)=1 .
$$

Thus $a a^{=}\left[\left(1-a a^{=} c u^{-}\left(1-a a^{=}\right]+c u^{-}\left(1-a a^{=}\right)=1\right.\right.$, which on multiplying through by

$$
p=\left[1+a a^{=} c u^{-}\left(1-a a^{=}\right)\right]\left(a^{=}\right)^{-1}
$$

yields:

$$
a+c t=p=\text { unit, where } t=u^{-}\left(1-a a^{=}\right)\left(a^{=}\right)^{-1} .
$$

Now if in addition, $a c=c a$ and $a^{=} c=c a^{=}$then we may take $u^{-}=c^{\sharp}$. Hence

$$
a+\left(1-a a^{=}\right)\left(a^{=}\right)^{-1} c^{\sharp} c=p
$$

implying that $R a+R c=R$. 
4. It is now clear how to extend this to the following: If $a^{k}$ is unit regular for some $k \geqq 1$, say $a^{k}\left(a^{k}\right)^{=} a^{k}=a^{k}$, where $\left(a^{k}\right)=$ is a unit, and if $c^{d}$ exist, such that $c c^{d}$ commutes with $a^{k}\left(a^{k}\right)=$ and $\left(a^{k}\right)=$ then

$$
R=a R+c R \Longrightarrow R=R a+R c .
$$

The case where $a^{d}$ exists and $a c=c a$ easily follows from this example because then $\left(a^{k}\right)^{*}$ exist, for some $k \geqq 1$ and one may then take $\left(a^{k}\right)^{=}=$ $\left(a^{k}\right)^{\sharp}+\left(1-a a^{d}\right)$.

\section{REFERENCES}

[1] G. Azumaya, Strongly-I-regular rings, J. Fac. Science Hokkaido Univ. Series 1, Math., XIII (1954-57), 34-39.

[2] A. Ben Israel and T. N. E. Greville, Generalized Inverses, Theory and Applications, Wiley, New York, 1974.

[3] S. L. Campbell, C. D. Meyer and N. J. Rose, Applications of the Drazin inverse to linear systems of differential equations, SIAM J. Appl. Math., 31 (1976), 411-425.

[4] M. P. Drazin, Pseudo-inverses in associate rings and semigroups, Amer. Math. Monthly, 65 (1958), 506-514.

[5] F. R. Gantmacher, The Theory of Matrices, Vol. 1, Chelsea, New York, 1960.

[6] R. E. Hartwig, Block generalized inverses, Arch. Rat. Mech., 61, (1976), 197-251.

[7] R. E. Harwig and J. Schoaf, Group inverses and Drazin inverses of bidiagonal and triangular Toeplitz matrices, J. Austral. Math. Soc. (A), XXIV (1977), 10-34.

[8] R. E. Hartwig and J. Luh, On finite regular rings, Pacific J. Math., 69 (1977), $73-95$.

[9] M. Henriksen, On a class of regular rings that are elementary divisor rings, Arch. Math., 24 (1973), 133-141.

[10] I. N. Herstein and L. W. Small, An extension of a theorem of Schur, Lin. Mult. Algebra, 3 (1975), 41-43.

[11] J. Lambek, Lectures on Rings and Modules, Blaisdell, Waltham, 1966.

Received June 2, 1977 and in revised form December 7, 1977.

North Carolina State University

RALEIGH, NC 27607 


\section{PACIFIC JOURNAL OF MATHEMATICS}

\section{EDITORS}

RICHARD ARENS (Managing Editor)

University of California

Los Angeles, California 90024

C. W. CurTis

University of Oregon

Eugene, OR 97403

C. C. MOORE

University of California

Berkeley, CA 94720
J. DUGUNDJI

Department of Mathematics University of Southern California Los Angeles, California 90007

R. FinN AND J. Milgram Stanford University Stanford, California 94305

\section{ASSOCIATE EDITORS}

E. F. BECKENBACH

B. H. NeumanN

F. WOLF

K. YoSHIDA

\section{SUPPORTING INSTITUTIONS}

UNIVERSITY OF BRITISH COLUMBIA CALIFORNIA INSTITUTE OF TECHNOLOGY UNIVERSITY OF CALIFORNIA MONTANA STATE UNIVERSITY UNIVERSITY OF NEVADA, RENO NEW MEXICO STATE UNIVERSITY OREGON STATE UNIVERSITY UNIVERSITY OF OREGON
UNIVERSITY OF SOUTHERN CALIFORNIA

STANFORD UNIVERSITY

UNIVERSITY OF HAWAII

UNIVERSITY OF TOKYO

UNIVERSITY OF UTAH

WASHINGTON STATE UNIVERSITY

UNIVERSITY OF WASHINGTON 


\section{Pacific Journal of Mathematics \\ Vol. 78, No. $1 \quad$ March, 1978}

Simeon M. Berman, A class of isotropic distributions in $\mathbf{R}^{n}$ and their

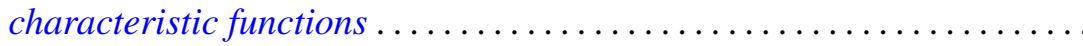

Ezra Brown and Charles John Parry, The 2-class group of biquadratic fields.

II ........................................ 11

Thomas E. Cecil and Patrick J. Ryan, Focal sets of submanifolds ....... 27

Joseph A. Cima and James Warren Roberts, Denting points in $B^{p} \ldots \ldots \ldots 41$

Thomas W. Cusick, Integer multiples of periodic continued fractions . . . . . 47

Robert D. Davis, The factors of the ramification sequence of a class of

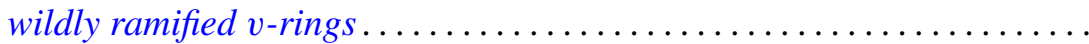

Robert Martin Ephraim, Multiplicative linear functionals of Stein

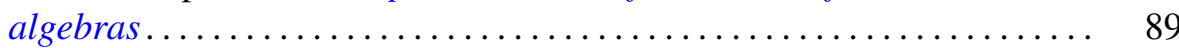

Philip Joel Feinsilver, Operator calculus . .................... 95

David Andrew Gay and William Yslas Vélez, On the degree of the splitting field of an irreducible binomial ..........................

Robert William Gilmer, Jr. and William James Heinzer, On the divisors of

monic polynomials over a commutative ring ..................

Robert E. Hartwig, Schur's theorem and the Drazin inverse .............

Hugh M. Hilden, Embeddings and branched covering spaces for three and four dimensional manifolds ............................

Carlos Moreno, The Petersson inner product and the residue of an Euler product. ...

Christopher Lloyd Morgan, On relations for representations of finite groups....

Ira J. Papick, Finite type extensions and coherence

$\mathrm{R}$. Michael Range, The Carathéodory metric and holomorphic maps on a class of weakly pseudoconvex domains ................

Donald Michael Redmond, Mean value theorems for a class of Dirichlet series

Daniel Reich, Partitioning integers using a finitely generated semigroup ...

Georg Johann Rieger, Remark on a paper of Stux concerning squarefree

numbers in non-linear sequences

Gerhard Rosenberger, Alternierende Produkte in freien Gruppen ..

Ryōtarō Satō, Contraction semigroups in Lebesgue space

Tord Sjödin, Capacities of compact sets in linear subspaces of $\mathbf{R}^{n}$

Robert Jeffrey Zimmer, Uniform subgroups and ergodic actions of exponential Lie groups......................... 